\title{
Triplex-forming DNA Probe Approach for Silver Detection and the Effect of C-G.C Triplet Distribution on Triplex Stability
}

Osman Doluca

Izmir University of Economics, Biomedical Engineering, Izmir, Turkey

\section{ABST RACT}

T $\mathrm{n}$ this study novel triplex forming DNA probes have been designed in order to detect Ag+ ion in low concentrations. The use of triplex forming oligonucleotides is a convenient in applications of sensing biomolecules due to their sequence specificity and programmability. However, the use of triplexes has its own obstacles. While antiparallel triplex forming sequences tend to prefer G-quadruplex formation over triplexes, parallel triplexes are also challenging because their formation is triggered by lowering the $\mathrm{pH}$, or using of high concentrations of cations for the stabilization of C-G.C triplets, ie. $\mathrm{Ag}^{+}$. While due to electrostatic forces C-G.C triplets stabilize in the presence of cations, this limits possible choices for a triplex forming sequence. A better understanding of the impact of the sequence and designing accordingly may improve the stability of a triplex and lower the need for high cation concentration. Here we have present Triplex-forming DNA-based probes with different distributions of C-G.C triplets for detection of Ag+ and show the impact of the C-G.C triplet distribution on the stability of parallel triplexes. Our results indicate Ag+ detection as low as $20 \mathrm{nM}$ and show dramatic increase in stability when C-G.C triplets are positioned at the flanks of the triplex.

\section{Keywords:}

Parallel triplex DNA; Triple helical DNA; Triplex DNA; DNA topology, Ag; Silver.

\author{
Article History: \\ Received: 2019/10/30 \\ Accepted: 2019/12/04 \\ Online: 2019/12/31
}

Correspondence to: Osman Doluca, Izmir University of Economics, Biomedical Engineering, lzmir, TURKEY

E-Mail: osman.doluca@ieu.edu.trPhone: $+902324888272$

\section{INTRODUCTION}

B esides the knowledge that DNA is the primary hereditary molecule with possessing genetic code hidden in its double stranded helix form (B-form), an increasing number of evidence demonstrate that DNA, due to its non-canonical (non-B-form) structures, have regulatory functions on various cellular processes as well [1]. While duplex form of DNA is built up via Watson-Crick base pairing edges of nucleotide residues, some forms of the DNA are created by binding of auxiliary strands to a duplex DNA via Hoogsten base pairing edges of nucleotides [2,3]. In particular, triplex DNA forms have become one of the primary research areas due to the possibility of targeting regulatory regions of several oncogenes [24] and that their providing ease to manipulation due to exhibiting high stability in comparison to duplex forms [5].

These triplex DNA forms could be created via binding of an auxiliary strand which is either rich in purine or pyrimidine, purine rich strand of the duplex DNA [6].
Depending on the orientation of the auxiliary strand versus the purine rich strand of the duplex, these DNA forms could be created in a parallel fashion, possessing $\mathrm{C}^{+} \cdot \mathrm{G}-\mathrm{C}$ and $\mathrm{T} \cdot \mathrm{A}-\mathrm{T}$ triplets or antiparallel fashion possessing G•G-C and A•A-T triplets (• indicates Hoogsten base pairing of third strand, - indicates Watson-Crick base pairing between two strands of duplex DNA) [7]. Despite the fact that both parallel and antiparallel triplex DNA forms could be created in physiological environment, $\mathrm{C}^{+} \cdot \mathrm{G}-\mathrm{C}$ triplexes require protonation of cytosine residues of third strand at the position of N3. Therefore, particularly low $\mathrm{pH}$ values enable formation of $\mathrm{C}^{+} \cdot \mathrm{G}-\mathrm{C}$ triplets and associated parallel triplexes [8] Furthermore, there are many characteristics affecting stability of triplexes, such as complementarity and base distribution. A random distribution of purine residues on the duplex sequence does help binding of the third strand and higher stability could be achieved via stacking of these residues consecutively [5]. In the light of these information triplex DNA forms are frequently investigated and manipulated in biotechnological applica- 
tions. For instance the use of triplex DNA as probes is one of promising applications [9]. In this scope, many triplex DNA probes have been designed recently detecting oligonucleotide molecules with sequence specificity such as those including mutations in their sequence [10] and heavy metals that cause health issues around the globe [11].

The main efforts on triplex DNA structures are focussed on increasing stability of both parallel and antiparallel triplexes as well as to achieve $\mathrm{C}^{+} \cdot \mathrm{G}-\mathrm{C}$ triplexes at neutral $\mathrm{pH}$ values. There are many studies applying chemical modifications on nucleotide residues and redesign the backbone of DNA molecules in literature [12]. As such Ihara and his colleagues have demonstrated that $\mathrm{Ag}^{+}$stabilized the $\mathrm{C}^{+} \bullet \mathrm{G}-\mathrm{C}$ motif via displacing N3 proton of cytosines. Therefore they have achieved parallel $\mathrm{C}^{+} \cdot \mathrm{G}-\mathrm{C}$ triplexes at neutral $\mathrm{pH}$ values without protonation of cytosine residues enabling alternative [13].

Silver ion $\left(\mathrm{Ag}^{+}\right)$has reached wide utilization area from jewelry designing [14] to photography [15] and electronic industry [16] over the years due to its prominent characteristics such as high thermal [17] and electrical conductivity [18], contact resistance [19] etc. Furthermore, due to its high antibacterial [20] and antifungal characteristics [20,21], silver ion is frequently used in medical applications as well, such as production of bone prostheses [22], dental amalgams [23], and catheters [24]. Besides, silver ion could be used as a therapeutic agent in the treatment of many other disorders, for instance mental illness, epilepsy, infectious diseases, nicotine addiction etc [25].

On the other hand, exposure to high amounts of silver through ingestion, inhalation, and skin contact may also cause serious illnesses. Silver is attributed as bioactive compound as it could form protein complexes once it enters the human body through any exposure route. It's discovered that this bioactivity serve a toxic profile of the silver ion which could cause various hazardous disorders in human body. [26] Most studied and prevalent diseases by high exposure of silver ion are called argyria and argyrosis which are characterized as pigment disorders on skin and eyes, respectively. Symptoms of these diseases are observed as turning color of exposure areas into ash gray [27,28]. Additionally, that silver accumulates among many tissues such as kidney, liver, skin, and spleen when high exposure of silver is observed is another fact affecting human health. This accumulation causing impairment over various pathways on related tissue indicates high toxicity of silver ion. [29] For instance it's revealed that high amount of silver causes to structural and functional disorders on red blood cells by depleting of reduced glutathione that is required for biochemical reactions. As such permissible exposure limit (PEL) of both metallic and soluble silver ion was determined as $0.01 \mathrm{mg} / \mathrm{m}^{3}$ by the
Mine Safety and Health Administration (MSHA) and the Occupational Safety and Health Administration (OSHA) in the past decades. [26] Thereby, the detection of silver concentration even in low values among food products, tap water, environment, and any product that could contact with body has been came into prominence when to consider its exposure routes and possible effects on human health.

In this study we report three novel triplex DNA probes (TP1, TP2, and TP3) with parallel C+•G-C motifs in order to detect silver ion concentration. Each triplex probes have been functionalized with fluorescein (FAM) and TAMRA molecules at 5 ' end and in the middle, respectively. The stabilization of the triplex formation via binding of $\mathrm{Ag}^{+}$gives rise to quenching of FAM strength of which depends on the ion concentration. Moreover, the effect of $\mathrm{C}^{+} \cdot \mathrm{G}-\mathrm{C}$ sequence alignment on the stability of triplex probes against temperature and urea was investigated. Comparison of the probe sequences also demonstrated that while our triplex DNA probes are efficient tools for detection of silver concentration even in low values, $\mathrm{C}^{+} \cdot \mathrm{G}-\mathrm{C}$ distribution has a significant effect on the stability of the triplex DNA forms, with little impact on the ion detection range.

\section{MATERIAL AND METHODS}

\section{Triplex DNA Probes Designs and Sample Preparations}

The three triplex-forming DNA probes have been designed with distinct C-G.C triplet distribution. TP1, TP2 and TP3, triplex-forming DNA sequences have been purchased with sequences of 5'-/FAM/CTC TCT CTC TCT TTT TCT CTC TCT CTC TTT/TAMRA/ TGA GAG AGA GAG A-3', 5'-/FAM/CTT CCT TCC CTT TTT TTC CCT TCC TTC TTT/TAMRA/ TGA AGG AAG GGA A-3' and 5'-/FAM/CCC TTC CTT CTT TTT TTC TTC CTT CCC TTT/TAMRA/ TGG GAA GGA AGA A-3', respectively. (Pentabase, Denmark) All sequences were dissolved in $10 \mathrm{mM}$ Tris-EDTA (TE) buffer at $\mathrm{pH}$ 7.4 with $100 \mu \mathrm{M}$ final sequence concentration. As a silver ion source, $\mathrm{AgNO}_{3}$ was dissolved in Tris-Acetate buffer at $10 \mathrm{mM}$ concentration. $\mathrm{No} \mathrm{HCl}$ was used during $\mathrm{pH}$ adjustment to avoid precipitation with silver. All solutions were stored in $4{ }^{\circ} \mathrm{C}$ until utilization in further analysis.

\section{Circular Dichroism (CD) Spectrophotometry Analysis}

The structural alterations of free triplex DNA probes with silver binding have been investigated with Jasco J-1500 circular dichroism (CD) spectrophotometer device. All CD measurements were performed at $0.1 \mu \mathrm{M}$ 


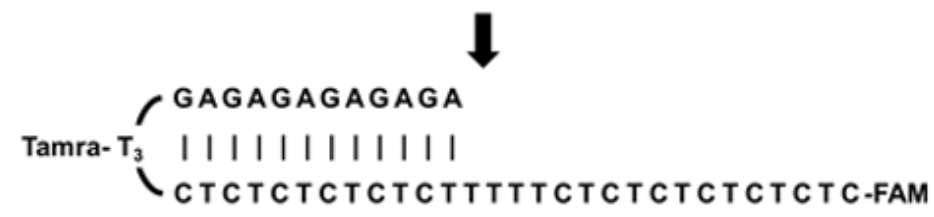

B)

$\downarrow \mathrm{Ag}^{+}$

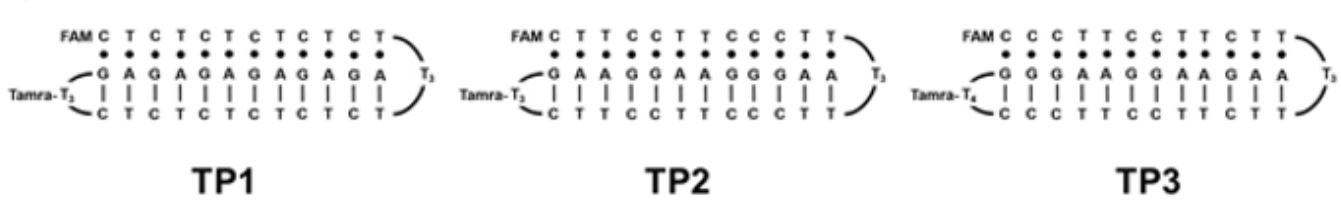

Figure 1. Silver mediated triplex formation (A) and triplex-forming probes used in this study (B).

probe concentration with or without $100 \mu \mathrm{M} \mathrm{Ag} \mathrm{Ag}^{+}$in buffer. Samples were analyzed between 200-350 $\mathrm{nm}$ and 300-700 nm wavelengths.

\section{Fluorescence Spectrophotometry Analysis}

FRET characteristic fluorescence change following silver binding of triplex DNA probes have been analysed by Jasco FP-8500 fluorescence spectrophotometer with Ag+ concentration set between 0.02 and $2.0 \mu \mathrm{M}$ in TE buffer. Triplex DNA probes were set to $0.1 \mu \mathrm{M}$. The emission spectra have been analysed between 500 and $700 \mathrm{~nm}$. All fluorescence measurements were performed using $5 \mathrm{~nm}$ slit sizes and $0.5 \mathrm{~nm}$ data pitch at $100 \mathrm{~nm} / \mathrm{min}$ data collection rate.

\section{Urea Denaturation Analysis}

Stability differentiation of triplex DNA probes against urea denaturation has been analyzed by Jasco FP-8500 fluorescence spectrometer. Samples containing $0.1 \mu \mathrm{M}$ DNA probe mixed with $120 \mu \mathrm{M} \mathrm{Ag}^{+}$in TE buffer has been incubated with different concentrations of urea ranging from $0 \mathrm{M}$ to $8 \mathrm{M}$. The samples had been excited at $485 \mathrm{~nm}$ and the emission of FAM was analyzed between 500-700 nm wavelength. Denaturation curves were constructed from the emission at $522 \mathrm{~nm}$.

\section{Annealing Temperature Analysis}

The annealing temperatures and the formation of the triplexes were analysed using fluorescence characteristics by Jasco FP-8500 fluorescence spectrophotometer. Samples containing $0.1 \mu \mathrm{M}$ DNA probes mixed with 120 $\mu \mathrm{M} \mathrm{Ag}^{+}$had been heated to $80^{\circ} \mathrm{C}$ and FAM emission was analyzed by cooling with constant scales until room temperature was reached. Samples were excited at $485 \mathrm{~nm}$ and emission values has been detected between 500-700 nm wavelengths. Renaturation curves were constructed from the emission at $522 \mathrm{~nm}$.

\section{RESULTS AND DISCUSSION}

Three intramolecular parallel triplex forming sequences were designed so that the second loop from the 5 ' end is required to fold to form a duplex and the first one to fold to form a triplex. The sequences bear a 5' FAM modification and an internal TAMRA modification attached to a thymine located in the second loop so that in unfolded form the distance should be around $10 \mathrm{~nm}$ which is too large for FRET to occur. The sequences are designed so that duplex formation alone should not decrease FAM and TAMRA distance except only upon triplex formation this distance would decrease allowing TAMRA quenching FAM fluorescence. The only differences between the three probe designs is at the sequence level, particularly the distribution of C-G.C triplet, while keeping overall counts of C-G.C triplets equal to 6. In TP1 C-G.C triplets are evenly distributed and separated by T-A.T triplets. In TP2 C-G.C triplets were concentrated to be located further away from the fluorescent moieties and the second loop and closer to the first loop which was responsible for triplex formation. In case of TP3, C-G.C triplets were located away from the first loop and closer to the fluorescent moieties. All loops were composed of four thymines (Fig. 1).

\section{Duplex Triplex Transition}

The triplex formation is expected to occur upon the folding of the first loops and controlled by $\mathrm{Ag}^{+}$concentration. FAM emission at $520 \mathrm{~nm}$ decrease was observed for all probes upon addition of $\mathrm{AgNO}_{3}$. For probe concentrations of $0.1 \mu \mathrm{M}$, this transition was apparent starting from as low as $20 \mathrm{nM}$ of $\mathrm{AgNO}_{3}$ until $2 \mu \mathrm{M}$. In comparison TAMRA emission became apparent at $580 \mathrm{~nm}$ as 
A)

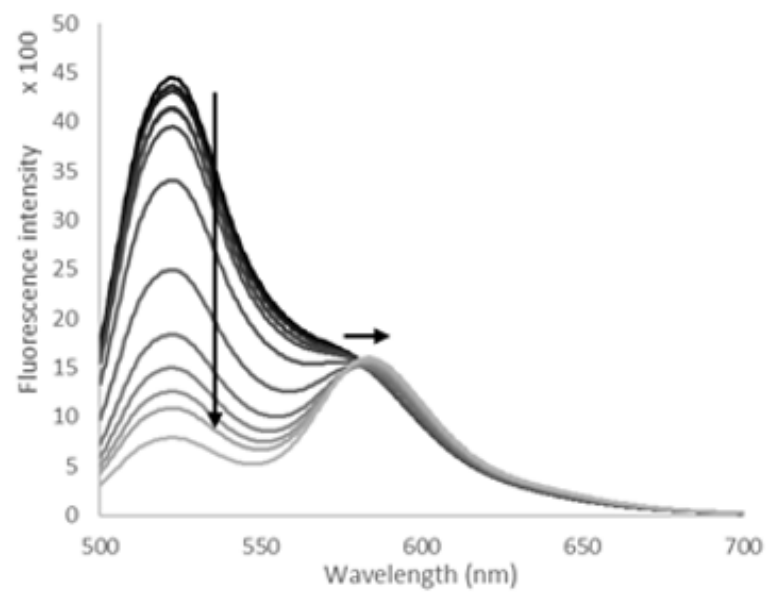

B)

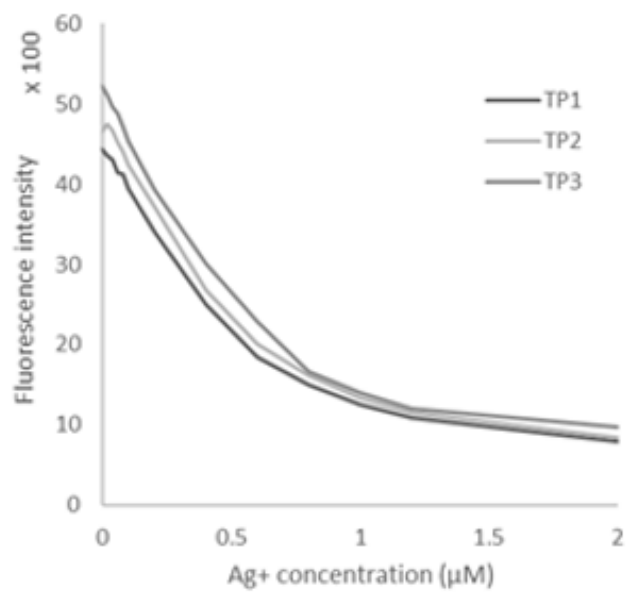

Figure 2. Change in fluorescence spectrum of TP1 (A) and fluorescence intensity of all probes (B) with increasing $\mathrm{Ag}^{+}$concentration.
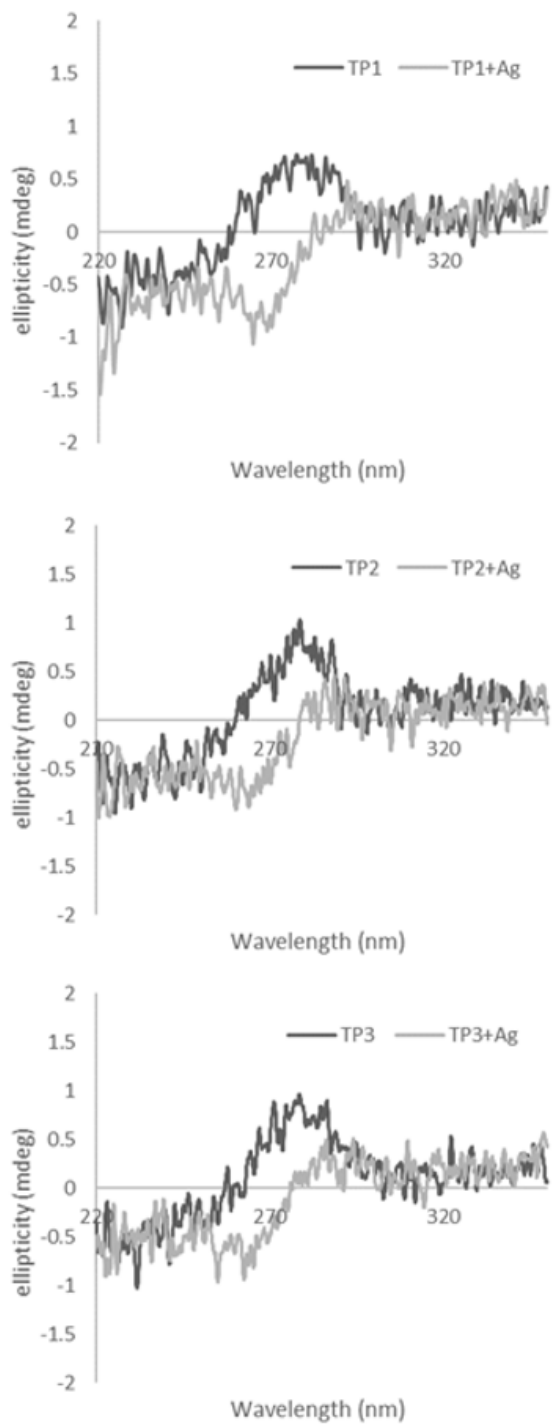

Figure 3. Change in circular dichroism spectra of the probes upon triplex formation.
FAM fluorescence decreased. The transition was sharp and linear between 0.1 and $0.8 \mu \mathrm{M}$. We observed little difference in fluorescence with $\mathrm{Ag}^{+}$titration of the probes. (Fig. 2) Structural transition was also confirmed by $\mathrm{CD}$ analysis where addition of $\mathrm{AgNO}_{3}$ dramatic change in CD signatures as the maxima at $280 \mathrm{~nm}$ of the duplex converts to a minima at $260 \mathrm{~nm}$. At the same time, no significant CD signature was detected above $300 \mathrm{~nm}$. It should be noted that the CD signals were quite low due to low probe concentrations (Fig. 3).

\section{Difference in Melting and Annealing}

Although all probes showed highly similar characteristics upon $\mathrm{AgNO}_{3}$ addition, they differed in terms of their stability. Urea mediated denaturation showed that while TP1 and TP2 showed similar denaturation curves with a maximum change between 0 and 1 M Urea, TP3 showed significantly better stability. TP3 denaturation showed a longer transition period starting from $0 \mathrm{M}$ to $3 \mathrm{M}$. The improved stability of TP3 was also apparent in case of annealing studies. When probes melt at elevated temperatures were allowed to cool down and form triplex in the presence of $\mathrm{Ag}^{+}$, the annealing temperature (Ta) was found as the temperature where emission change was maximum. It was shown that $32^{\circ} \mathrm{C}$ was the $\mathrm{T}_{\mathrm{a}}$ for both TP1 and TP2, while for TP3 Ta was $62^{\circ} \mathrm{C}$ (Fig. 4).

For all three probes tested here, $\mathrm{Ag}^{+}$mediated parallel triplex formation was demonstrated. $\mathrm{Ag}^{+}$titration decreased FAM fluorescence while TAMRA showed slight increase in fluorescence, indicating FRET between FAM and TAMRA which is explained by decreased distance between the fluorophores. The decreased distance is expected to occur upon folding of the first loop as a result of parallel triplex formation. Alternatively it may be possible upon association 

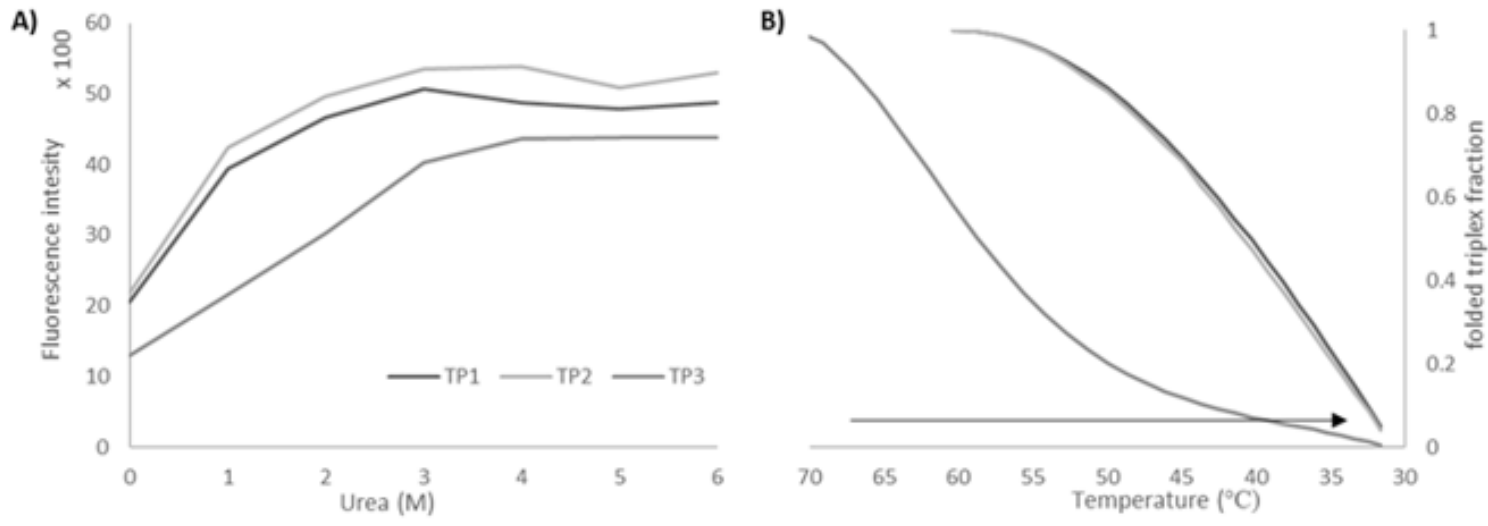

Figure 4. Urea denaturation (A) and annealing (B) analysis of the probes used in the study.

of two probes to form a bimolecular parallel triplex, however, it is less likely, especially in lower concentrations of the probes. The structural difference was also shown via circular dichroism at around 250 and $280 \mathrm{~nm}$ where the signal may only dependent on the nucleobases, indicating a structural transition at the DNA level.

While the impact of the distribution of the C-G.C triplets was not apparent for their affinity towards Ag+, it became clear with the stability tests. The melting of a helical structure, whether it be duplex or triplex, is expected to start from the ends. In the case of the triplex-forming probes used here, the 3 ' end of the triplex-forming branch is stabilized by the loop, leaving the 5 ' end as the meltingstart site. Comparing the triplex-forming probes, with three C-G.C triplets located at the 5' end in TP3 is expected to benefit its stability over others, through the stabilising effect of the Ag+ on the C-G.C triplets. Indeed, on both urea denaturation and annealing studies TP3 showed significantly better stability. In comparison, TP1 and 2, which have only one C-G.C triplets at the 5' end, showed marginal difference between each other.

\section{CONCLUSION}

Here we have showed triplex forming probes with different C-G.C distributions are equally effective in detection of Ag+. While $\mathrm{Ag}^{+}$stabilized C-G.C triplets that are located at the ends of the sequence does not affect the affinity towards the $\mathrm{Ag}^{+}$, it contributes to the overall stability of the triplexes. In the light of our findings we advise inclusion of three C-G.C triplets at the flanks of parallel triplex-forming sequences whether it be stabilized by $\mathrm{Ag}^{+}$or $\mathrm{H}^{+}$for improved overall stability. With either cation the C-G.C triplets would contribute to the stability more than T-A.T triplets due to the additional electrostatic forces besides the H-bonds. Furthermore, in the light of knowledge about disorders basing on $\mathrm{Ag}^{+}$toxicity, the importance of $\mathrm{Ag}^{+}$detection was emphasized previously. Our results demonstrated that the triplex formings DNA probe could be a novel biotechnological approach to detect $\mathrm{Ag}^{+}$even in low concentrations between the range of 0.02-2.0 $\mu \mathrm{M}$.

\section{ACKNOWLEDGEMENT}

This study was supported by Izmir University of Economics (BAP 2017-05).

\section{References}

1. Pugeta N, Miller KM, Legube G. Non-canonical DNA/RNA structures during Transcription-Coupled Double-Strand Break Repair: Roadblocks or Bona fide repair intermediates? DNA Repair (2019) 102661.

2. Travers A, Muskhelishvili G. DNA structure and function. FEBS Journal 282 (2015) 2279-2295.

3. Frank-Kamenetskii MD, Mirkin SM. Triplex DNA structures. Annual Reviews of Biochemistry 64 (1995) 65-95.

4. Christensen LA, Finch RA, Booker AJ, Vasquez KM. Targeting oncogenes to improve breast cancer chemotherapy. Cancer Research 66 (2006) 4089-4094.

5. Rusling DA. The stability of triplex DNA is affected by the stability of the underlying duplex. Biophysical Chemistry 145 (2009) 105110 .

6. Maldonado R, Filarsky M, Grummt I, Längst G. Purine- and pyrimidine-triple-helix-forming oligonucleotides recognize qualitatively different target sites at the ribosomal DNA locus. RNA 24 (2018) 371-380

7. Jain A, Wang G, Vasquez KM. DNA triple helices: biological consequences and therapeutic potential. Biochimie 90 (2008) 11171130 .

8. Bacolla A, Wang G, Vasquez KM. New Perspectives on DNA and RNA Triplexes As Effectors of Biological Activity. PLoS Genetics 11 (2015) e1005696.

9. Hu Y, Cecconello A, Idili A, Ricci F, Willner I. Triplex DNA Nanostructures: From Basic Properties to Applications. Angewandte Chemie International Edition 56 (2017) 15210-15233.

10. Yang Y, Huang Y, Li C. A reusable electrochemical sensor for one-step biosensing in complex media using triplex-forming oligonucleotide coupled DNA nanostructure. Analytica Chimica Acta 1055 (2019) 90-97.

11. Ma DL, Ma VPY, Chan DSH, Leung KH, He HZ, Leung CH. Recent advances in luminescent heavy metal complexes for 
sensing. Coordination Chemistry Reviews 256 (2012) 3087-3113 doi:10.1016/j.ccr.2012.07.005

12. Torigoe H, Nakagawa O, Imanishi T, Obika S, Sasaki K. Chemical modification of triplex-forming oligonucleotide to promote pyrimidine motif triplex formation at physiological $\mathrm{pH}$. Biochimie 94 (2012) 1032-1040.

13. Ihara T, Ishii T, Araki N, Wilson AW, Jyo A. Silver ion unusually stabilizes the structure of a parallel-motif DNA triplex. Journal of the American Chemical Society 131 (2009) 3826-3827.

14. Aktepe N, Kocyigit A, Yukselten Y, Taskin A, Keskin C, Celik H. Increased DNA damage and oxidative stress among silver jewelry workers. Biological Trace Element Research 164 (2015) 185-191.

15. Villena AN. Exploring confocal microscopy to analyze ancient photography. Journal of Cultural Heritage 36 (2019) 191-199.

16. Zhang H, Suganuma K. Sintered Silver for LED Applications, in: Siow KS (Ed) Die-Attach Materials for High Temperature Applications in Microelectronics Packaging. Springer, pp 35-65, 2019. doi:10.1007/978-3-319-99256-3_2.

17. Abbasi S. The thermal conductivity modeling of nanofluids involving modified $\mathrm{Cu}$ nanorods by $\mathrm{Ag}$ nanoparticles. Heat and Mass Transfer 55 (2019) 891-897. doi:10.1007/s00231-018-2476-2.

18. Sun G, Wang Z, Huang J. Electromagnetic shielding effectiveness and electrical conductivity of a thin silver layer deposited onto cellulose film via electroless plating. Journal of Materials Science: Materials in Electronics 30 (2019) 12044-12053. doi:10.1007/ s10854-019-01562-z.

19. Chung S, Jeong J, Kim D, Park Y, Lee C, Hong Y. Contact Resistance of Inkjet-Printed Silver Source-Drain Electrodes in BottomContact OTFTs. Journal of Display Technology 8 (2012) 48-53.

20. Sarkar R. Aqueous synthesis and antibacterial activity of Silve nanoparticles against pseudomonas putida. Materials Today: Proceedings 11 (2019) 686-694.

21. Bocate KP. Antifungal activity of silver nanoparticles and simvastatin against toxigenic species of Aspergillus. International Journal of Food Microbiology 291 (2019) 79-86.

22. Streitbuerger A, Henrichs MP, Hauschild G, Nottrott M, Guder W, Hardes J. Silver-coated megaprostheses in the proximal femur in patients with sarcoma. European Journal of Orthopaedic Surgery \& Traumatology 29 (2019) 79-85

23. Mohammadi Z, Mesgar AS, Rahmdar S, Farhangi E. Effect of setting time and artificial saliva on the strength evaluated by different methods of dental silver amalgam: A comparative study. Materialwissenschaft Und Werkstofftechnik 50 (2019) 747-760. doi:10.1002/mawe.201800022.

24. Akcam FZ, Kaya O, Temel EN, Buyuktuna SA, Unal O, Yurekli VA. An investigation of the effectiveness against bacteriuria of silver-coated catheters in short-term urinary catheter applications: A randomized controlled study. Journal of Infection and Chemotherapy 25 (2019) 797-800. doi:10.1016/j.jiac.2019.04.004.

25. Gulbranson SH, Hud JA, Hansen RC. Argyria following the use of dietary supplements containing colloidal silver protein. Cutis 66 (2000) 373-374.

26. Drake PL, Hazelwood KJ. Exposure-Related Health Effects of Silver and Silver Compounds: A Review. Annals of Occupational Hygiene 49 (2005) 575-585.

27. Molina-Hernandez AI, Diaz-Gonzalez JM, Saeb-Lima M, Dominguez-Cherit J. Argyria after Silver Nitrate Intake: Case Report and Brief Review of Literature. Indian Journal of Dermatology 60 (2015) 520.

28. Pala G, Fronterré A, Scafa F, Scelsi M, Ceccuzzi R, Gentile E, Candura SM. Ocular argyrosis in a silver craftsman. Journal of Occupational Health 50 (2008) 521-524.

29. Lansdown ABG. A pharmacological and toxicological profile of silver as an antimicrobial agent in medical devices. Advances in Pharmacological Science 2010 (2010) 910686. 